\title{
Deletion of p38a MAPK in microglia blunts trauma-induced inflammatory responses in mice
}

\author{
Josh M. Morganti ${ }^{1,2,3}$, Danielle S. Goulding ${ }^{1}$ and Linda J. Van Eldik ${ }^{1,2,3^{*}}$
}

\begin{abstract}
Traumatic brain injury (TBI) is a significant cause of morbidity and mortality in the USA and other developed countries worldwide. Following the initial mechanical insult, the brain's primary innate immune effector, microglia, initiate inflammatory signaling cascades and pathophysiological responses that can lead to chronic neuroinflammation and neurodegenerative sequelae. The p38a MAPK signaling pathway in microglia is a key contributor to inflammatory responses to diverse disease-relevant stressors and injury conditions. Therefore, we tested here whether microglia p38a contributes to acute and persistent inflammatory responses induced by a focal TBI. We generated conditional cell-specific knockout of p38a in microglia using a CX3CR1 Cre-lox system, subjected the p38a knockout and wild-type mice to a controlled cortical impact TBI, and measured inflammatory responses at acute (1-day) and subacute (7-day) post-injury time points. We found that deletion of p38a in microglia only was sufficient to attenuate multiple pro-inflammatory responses following TBI, notably reducing pro-inflammatory cytokine/chemokine production and recruitment of inflammatory monocytes into the brain and preventing the persistent microglial morphological activation. These data provide strong evidence supporting a role for microglial p38a in propagation of a chronic and potentially neurotoxic pro-inflammatory environment in the brain following TBI.
\end{abstract}

Keywords: TBI, p38a, Kinase, Microglia, Monocytes, Cytokines, Chemokines, Neuroinflammation, Knockout

\section{Introduction}

Following TBI, the innate immune system is activated to induce the recruitment of microglia to the site of injury $[1,2]$, followed by the subsequent recruitment and infiltration of multiple waves of systemic immune mediators [3-6]. Presumably, these conserved tissue responses [7] are geared to protect and repair the brain from further injury. However, TBI can also lead to the persistent activation of microglia for many years following the initial TBI [8-10], and these secondary inflammatory responses can contribute to neurodegenerative sequelae [9-11].

A well-established signaling pathway that responds to a variety of inflammatory stressors is the p38 mitogen-activated protein kinase (MAPK) pathway,

\footnotetext{
* Correspondence: linda.vaneldik@uky.edu

${ }^{1}$ Sanders-Brown Center on Aging, University of Kentucky, 101 Sanders-Brown Bldg., 800 S. Limestone Street, Lexington, KY 40536, USA

${ }^{2}$ Department of Neuroscience, University of Kentucky, Lexington, KY, USA

Full list of author information is available at the end of the article
}

especially the $\mathrm{p} 38 \alpha$ isoform [12]. Although $\mathrm{p} 38 \alpha$ is expressed in virtually all cell types and tissues, it can have a variety of roles in development, homeostasis, and disease mechanisms. We have shown that $\mathrm{p} 38 \alpha$ in microglia is a key component regulating the production of cytokines in response to a variety of disease-related stimuli in cell and animal models. For example, our previous findings using the LysM-Cre model of $\mathrm{p} 38 \alpha$ deletion showed a robust suppression of trauma-induced inflammatory responses and protection against synaptic protein loss and vestibulomotor deficits [13]. However, a recent study [14] using the LysM-Cre system showed that LysM-driven recombination is not specific to only myeloid cell populations, but that there is a significant expression in neurons. Given the ubiquitous nature of p $38 \alpha$ and recent reports of effects of neuronal p38 $\alpha$ in animal models of degenerative disease [15-18], we sought to test more specifically the role of $p 38 \alpha$ in microglia following focal TBI. 
Therefore, we generated a more microglia-specific p38 $\alpha$ conditional knockout mouse, driven by the CX3CR1-Cre that can be used to selectively delete p38 $\alpha$ from the brain's resident immune cell, microglia. We tested if microglia p38 $\alpha$ is a significant contributor to TBI-induced neuroinflammatory responses, including the production of pro-inflammatory cytokines and recruitment of peripheral monocytes into the brain. We report here that loss of p38 $\alpha$ in microglia significantly reduces TBI-induced neuroinflammatory responses at both acute (1-day) and subacute (7-day) time points after injury.

\section{Methods}

\section{Animals}

All procedures involving animals for this study were approved by the Institutional Animal Care and Use Committee of the University of Kentucky. Mice were group housed in environmentally controlled conditions and provided food and water ad libitum.

To generate a cell-specific reporter line for validation of tamoxifen-induced recombination, we crossed homozygous CX3CR1 ${ }^{\text {CreERT2 }}$ mice [19] (Jax \# 020940) with


Resultant heterozygous mice, $A i 9^{\triangle C X 3 C R 1 C r e E R T 2}$, were used to measure tdTomato (RFP) expression in the brain myeloid fraction and in circulating peripheral blood mononuclear cells (PBMCs), following tamoxifen induction, as described below.

To generate the microglia-specific conditional knockout mice, we used our $\mathrm{p} 38 \alpha^{\mathrm{fl} / \mathrm{fl}}$ mice [13] crossed with CX3CR1 ${ }^{\text {CreERT2 }}$ mice [19] (Jax \# 020940) to generate hemizygous CX3CR1 ${ }^{+/ C r e E R T 2} \mathrm{p} 38 \alpha^{+/ f l}$ mice. Hemizygous carriers were mated to $\mathrm{p} 38 \alpha^{\mathrm{fl} / \mathrm{fl}}$ mice to generate $\mathrm{CX} 3 \mathrm{CR} 1^{+/ C r e E R T 2} \mathrm{p} 38 \alpha^{\mathrm{fl} / \mathrm{fl}}$ mice (KO, knockout). To generate experimental cohorts, we mated the $\mathrm{KO}$ mice back to $\mathrm{p} 38 \alpha^{\mathrm{fl} / \mathrm{fl}}$ mice to generate a roughly 50:50 distribution of $\mathrm{Cre}^{+} \mathrm{p} 38 \alpha^{\mathrm{fl} / \mathrm{fl}}(\mathrm{KO})$ and $\mathrm{Cre}^{\text {neg }} \mathrm{p} 38 \alpha^{\mathrm{fl} / \mathrm{fl}}$ (WT, wildtype) littermates. All mice were genotyped by Transnetyx. For all experiments, both male and female mice were used in a 50:50 ratio.

$A i 9^{\triangle C X 3 C R 1 C r e E R T 2}, \mathrm{WT}$, and $\mathrm{KO}$ mice, 2 months old, were fed tamoxifen in chow (Harlan, \#TD.130860, 400 ppm) for 28 days [19] to initiate Cre-mediated recombination in the $\mathrm{KO}$ mice as well as control for any confounds due to tamoxifen exposure in the WT mice. Following this period, there was a 28-day washout period wherein mice were fed standard diet, yielding experimental mice that were approximately 4 months old. The washout period was used because this Cre + mouse line has been shown to equally affect both microglia and bone marrow-derived mononuclear cells following tamoxifen administration [19]. However, following at least a 2-week washout of tamoxifen, peripheral mononuclear cells are replaced with non-recombined cells [19], whereas the recombined microglia persist.

\section{Surgery}

Approximately 4-month-old WT and p38 $\alpha$ KO mice underwent surgery for moderate focal TBI using the controlled cortical impact (CCI) method to the right parietal lobe, as we have previously described [5, 20, 21]. Briefly, mice were anesthetized, heads shaved, and maintained with $2.5 \%$ isoflurane with a non-rebreathing nose cone and passive exhaust system connected to a stereotaxic frame (Stoelting). Once animals were secured with non-traumatic ear bars, eye ointment was applied, and their shaved heads were swabbed with betadine. A midline incision was made through the scalp. Mice received a craniectomy approximately $3.5 \mathrm{~mm}$ in diameter using an electric microdrill with the center point determined by a digitally calibrated manipulator arm (Leica) to the coordinates: anteroposterior, $-2.0 \mathrm{~mm}$; mediolateral, $+2.0 \mathrm{~mm}$, with respect to bregma. Explicit attention was paid to prevent damage to the dura during craniectomy; any animal in which the dura was disrupted, as assessed by excessive bleeding, was omitted from the study and replaced by another littermate. Following craniectomy, contusion was achieved using a $3.0-\mathrm{mm}$ convex tip attached to an electromagnetic impactor (Leica) mounted to the digitally calibrated manipulator arm. The manipulator arm was rotated $20^{\circ}$ on the vertical axis, to allow impact flush with the natural curvature of the head/tissue. The parameters for impact were for a contusion depth of $0.9 \mathrm{~mm}$ (from dura), velocity was constant at $4.0 \mathrm{~m} / \mathrm{s}$, and the impact was sustained for $300 \mathrm{~ms}$. Importantly, these injury parameters penetrated all layers of the cortex stopping short of disrupting the dorsal hippocampal structure (AP $-2.0 \mathrm{~mm}$; $\mathrm{ML}+2.0 \mathrm{~mm}$; DV $0.9 \mathrm{~mm}$ ). Following CCI injury, the scalp was stapled, and each mouse was placed on a heating pad until they fully recovered as exhibited by resumption of movement and grooming. Sham animals were treated to the above surgical parameters except that the CCI injury was omitted. Animals were randomly allocated to their respective endpoint analyses at either 1-day or 7-day post-surgery.

\section{qRT-PCR}

Following washout (described above) and euthanasia, enriched myeloid fraction was processed for RNA isolation using the RNeasy Plus mini kit (Qiagen \#74136) following the manufacturer's protocol. Eluted RNA was quantified using a NanoDrop 2000 (Thermo). Four hundred nanograms of eluted RNA was converted to cDNA using High Capacity cDNA Reverse Transcription Kit (Applied Biosystems \#4368813). Quantitative Real-Time PCR for gene expression was performed on a ViiA7 Real-Time PCR 




B

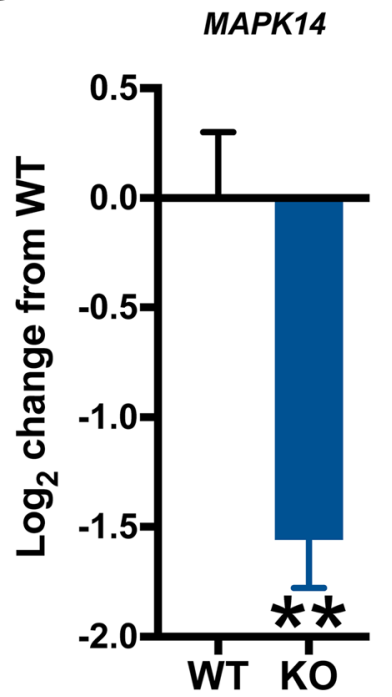

Fig. 1 Validation of conditional microglial knockout of p38a. a A cell-specific reporter (Ai9 ${ }^{\triangle C X 3 C R 1 C r e E R T 2}$ ) was used to validate the compartment restriction of tamoxifen-induced recombination. Aig ${ }^{\triangle C X 3 C R 1 C r e E R T 2}$ mice were placed on tamoxifen chow for 28 days, followed by a washout of 28 days on regular chow. In naïve mice, brain myeloid and blood PBMCs were examined for tdTomato (RFP) expression in tandem with CD11b (BUV395) expression. After the 28 day washout, $90.1 \%$ of CD11 b+ cells were labeled with RFP, whereas only $2.56 \%$ of circulating PBMCs were $\mathrm{RFP}^{+}$. b Myeloid-enriched fractions from naïve wild type (WT) and knockout (KO) mice were examined for p38a (MAPK14) gene expression following the tamoxifen washout procedures. Compared to WT mice, the p38a KO mice showed a significant reduction in p38a. Flow cytometry plots are representative of 3 separate experiments. For gene expression, $n=4$ per group. Data were analyzed using Student's $t$ test, ${ }^{*} p<0.05$. RFP red fluorescent protein, SSC side scatter

System (Applied Biosystems) using TaqMan gene expression assays for MAPK14 (Mm00442497_m1) and HPRT (Mm00446968_m1). Relative gene expression ratios were calculated using the $2^{-\Delta \Delta C T}$ method. All data were $\log _{2}$ transformed.

\section{MSD multiplex ELISA}

At the designated interval, mice were anesthetized with $2.5 \%$ isoflurane and transcardially perfused with ice-cold phosphate-buffered saline (PBS) for $5 \mathrm{~min}$. Following perfusion, the brains were rapidly removed, and the ipsilateral dorsal hippocampus was dissected and snap frozen in a 2-mL screw-top tube in liquid nitrogen. All dissected hippocampi were stored at $-80^{\circ} \mathrm{C}$ for subsequent biochemical evaluation. Hippocampi were processed for protein extraction using a high shear homogenizer (Omni TH115) using lysis buffer at a 1:10 weight to volume ratio. Tissue lysis buffer consisted of PBS containing $1 \mathrm{mM}$ PMSF and $1 \mathrm{mM}$ EDTA. Hippocampal homogenate was centrifuged at $12,000 \times g$ for 20 min at $4{ }^{\circ} \mathrm{C}$ in a Heraeus Megafuge 16R. Supernatants were collected for measurement of cytokines and chemokines using MesoScale Discovery (MSD) custom multiplex high-sensitivity (V-Plex) ELISA kits, as we have previously described [13].

\section{Flow cytometry}

For recombination validation using naïve $\mathrm{Ai} 9$ reporter mice, brains and blood from three sets of $A i 9^{\triangle C X 3 C R 1-}$ CrEERT2 mice were harvested following the $28 \mathrm{~d}$ tamoxifen washout period and used for myeloid cell and PBMC isolation, respectively. Brain myeloid cells were enriched using a discontinuous Percoll gradient (30:70), as others and we have previously described $[5,22]$. This method was also used in a naïve set of WT and KO mice to enrich myeloid cells from the brain to examine gene expression of p38 $\alpha$. Following centrifugation, the resultant myeloid enriched fraction at the 30:70 interface was aspirated and subsequently snap frozen prior to use for RNA harvesting and gene expression analyses.

PBMCs were isolated using diluted (1:3) fresh blood collected into plasma EDTA tubes, layered over FicollPaque+ (GE, \#17-1440-02). PBMCs were enriched using centrifugation at $1500 \times g$ for $20 \mathrm{mins}$ at $4{ }^{\circ} \mathrm{C}$. Cells were harvested and resuspended in FACS buffer (PBS + 0.5\% FCS) for staining. Brain myeloid cells and PBMC's were incubated with Zombie NIR (BioLegend, \#423106) followed by Fc block (Miltenyi \#130-092-575) and then anti-CD11b (BD \#565976).

For examining trauma-induced infiltration of monocytes at 1 day following injury, mice were anesthetized with $2.5 \%$ isoflurane and transcardially perfused with 


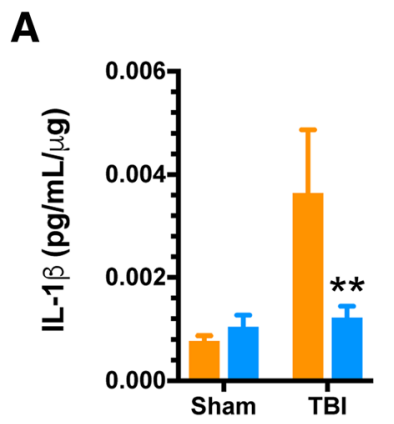

D

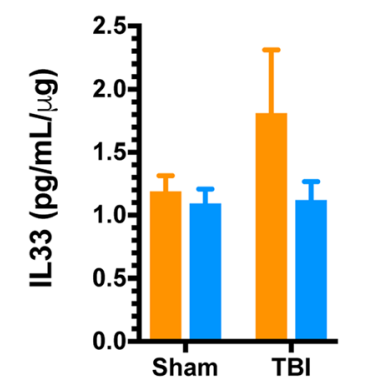

B

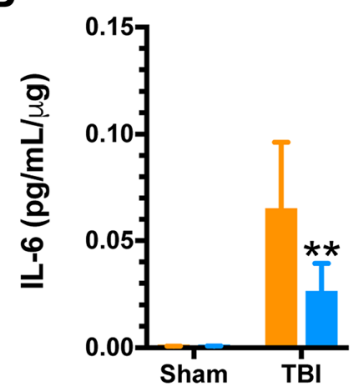

$\mathbf{E}$

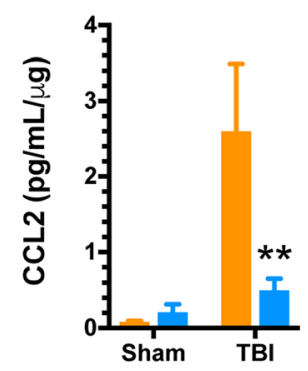

C

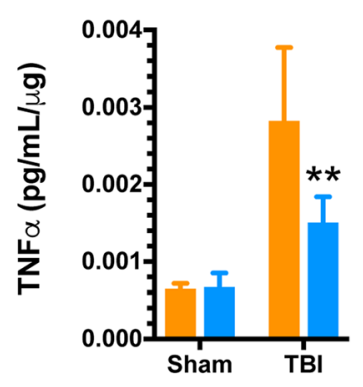

$\mathbf{F}$



Fig. 2 TBI-induced acute pro-inflammatory cytokine and chemokine up-regulation is mitigated by microglial p38a deficiency. Ipsilateral dorsal hippocampi were analyzed via high sensitivity multiplex MSD ELISA assay for pro-inflammatory cytokines and chemokines from sham and injured animals ( $n=8-10$ /group) at a 1 day post-injury interval. a-c The TBI-induced increases in pro-inflammatory cytokines IL-1 $\beta$, IL-6, and TNFa were significantly blunted, with $~ 50 \%$ lower levels in the p38a KO mice compared to WT mice. $\mathbf{d}$ The TBl-induced increase in IL-33 was also reduced in KO compared to WT mice, but this change was not significant. e,f The pro-inflammatory chemokines CCL2 and CXCL10 showed a marked reduction in the KO mice, compared to their injured WT counterparts. Data were analyzed using two-way ANOVA with Sidak's multiple comparison corrections on the pre-planned contrasts examining the effect of TBI in the WT versus KO conditions. ${ }^{*} p<0.05$, ${ }^{* *} p<0.01$, comparing $\mathrm{KO}$ (blue bars) to WT (orange bars)

ice-cold PBS for 5 min. Brains were rapidly removed and bisected, additionally removing the brainstem and cerebellum. The subsequent ipsilateral hemisphere was processed for myeloid cell enrichment using a discontinuous Percoll gradient, as described above. Following myeloid enrichment, cells were prepared for staining using Fc block (Miltenyi \#130-092-575). Cell-surface labeling was conducted using conjugated antibodies against CD11b (BD \#565976) and Ly6C (BD \#560594). Inflammatory monocytes were defined by this sequential gating strategy to enumerate the $\mathrm{CD}_{11} 1^{+} \mathrm{Ly} 6 \mathrm{C}^{+}$cell population. Cell viability was assessed using ZombieNIR (BioLegend \#423105). Spectral compensation was achieved using polystyrene microparticles (Miltenyi \#130-107-755) in combination with the above-listed antibodies following the manufacturer's suggested protocol. Cell analysis was conducted on a BD LSR II flow cytometer and analyzed using FlowJo software (Treestar, v10.0).

\section{Immunohistochemistry and analysis}

Using a sliding microtome with a freezing stage, we collected serial coronal sections $(30 \mu \mathrm{m})$ of the ipsilateral hemi-brain through the entire hemisphere and stored the sections in cryoprotectant at $-20^{\circ} \mathrm{C}$. Staining procedures were conducted on free-floating sections using every 12th section through the entire hemisphere. Primary and secondary antibodies were diluted in 3\% normal goat serum (LAMPIRE Biological Laboratories, catalog \#7332500) with $0.2 \%$ Triton X-100. Endogenous peroxidase activity was quenched with $3 \% \mathrm{H}_{2} \mathrm{O}_{2}$ in methanol, before the tissue blocking in $10 \%$ normal goat serum with $0.2 \%$ Triton X-100. Sections were incubated overnight at $4{ }^{\circ} \mathrm{C}$ with rabbit anti-Ibal $(1: 10,000$, Wako \#019-19741), followed by incubation with a HRP-conjugated goat anti-rabbit (Vector) secondary antibody. Subsequently, sections were developed in 0.5 $\mathrm{mg} / \mathrm{ml} \mathrm{3,3-diaminobenzidine} \mathrm{tetrahydrochloride} \mathrm{solution}$ (Sigma, catalog \#D5637). The tissue sections were dehydrated through gradients of ethyl alcohol and finally $x y-$ lene. The sections were coverslipped with Permount Mounting Medium (Fisher Scientific) and imaged on a Zeiss Axio Scan Z1 digital slide scanner at $\times 20$ magnification. Image analysis of the ipsilateral peri-contusion cortex and dorsal hippocampus, done by an investigator blinded to the study, used Halo analysis suite (Indica Labs, v2.3.2089.34) with the positive pixel algorithm; AreaQuantification v1.0. Iba1 positive staining was set to a baseline threshold on WT sham $\mathrm{Iba}^{+}$microglia pixel 



Fig. 3 TBI-mediated recruitment of inflammatory monocytes is significantly reduced in mice lacking microglial p38a. Using flow cytometric analyses, we quantified the proportional change in cellular infiltrate into the ipsilateral hemisphere of mice 1 day following injury. Inflammatory monocytes were defined by a sequential gating strategy to enumerate $\mathrm{CD} 11^{+} \mathrm{Ly} 6 \mathrm{C}^{+}$cell population in all four groups (WT and p38a KO, sham, and TBI). There were little-to-none inflammatory monocytes in the sham brains of either WT or KO mice. However, there was a robust increase observed in this cell population 1 day following TBI in WT mice, and this pathophysiological response was significantly blunted by 50\% in the KO mice. Data were analyzed using two-way ANOVA with Sidak's multiple comparison corrections on the pre-planned contrasts examining the effect of TBI in the WT versus KO conditions. ${ }^{* *} p<0.01$, comparing KO (blue bars) to WT (orange bars). SSC side scatter

intensity, such that any pixel at that intensity or greater (i.e., darker) was quantified as a pixel-positive area. The number of positive pixels was normalized per area outline for each section to account for outlined region-to-region area variability. All sections were batch analyzed using the same parameters.

\section{Statistics}

Statistical analyses were done using GraphPad Prism (v8). Pre-planned contrasts to examine the effect of TBI in WT versus $\mathrm{p} 38 \alpha \mathrm{KO}$ were conducted using two-way ANOVA with Sidak multiple comparison correction to examine pairwise response of TBI cohorts. Statistical significance was defined as $p<0.05$. All values are expressed as mean \pm SEM.

\section{Results}

\section{Microglial p38a knockout mitigates acute} neuroinflammatory responses to TBI

We validated the tissue restriction of the CX3CR1 ${ }^{\text {CreERT2 }}$ [19] line by crossing them to the Ai9 reporter line, inducing recombination with a 28-day induction with tamoxifen chow, and then using a 28-day washout period on standard chow to allow peripheral mononuclear cells to be replaced with non-recombined cells. As shown in Fig. 1a, this results in the overwhelming majority of tdTomato
(RFP) expression being observed in the brain $(90.1 \%$ of $\mathrm{RFP}^{+} \mathrm{CD}_{11 \mathrm{~b}^{+}}$cells in the brain versus $2.56 \%$ of $\mathrm{RFP}^{+}$PBMCs in the blood). Further, using this induction followed by washout paradigm, we also found a significant decrease in $\mathrm{p} 38 \alpha$ (MAPK14) gene expression in the enriched myeloid fraction from $\mathrm{p} 38 \alpha \mathrm{KO}$ mice compared to WT mice (Fig. 1b). These data document the microglial selectivity of the CX3CR1-Cre system and demonstrate a substantial decrease in $\mathrm{p} 38 \alpha$ expression in the $\mathrm{KO}$ mice.

We have previously shown that $\mathrm{p} 38 \alpha$ is an integral mediator of microglial inflammatory responses in vitro and in vivo [23]. Further, p38 $\alpha$ knockout in myeloid cells using the LysM-Cre model is neuroprotective following TBI [13]. However, because of recent data indicating significant expression in neurons with this LysM-Cre model system [14], it was important to test the effect of the more microglia-specific, CX3CR1-Cre mediated p38 $\alpha$ deletion on trauma-induced pro-inflammatory responses. To do this, we first examined the acute response of multiple neuroinflammatory analytes in WT and $\mathrm{p} 38 \alpha \mathrm{KO}$ mice at 1-day post-injury. As shown in Fig. 2, CCI induces a robust increase in multiple pro-inflammatory cytokines and chemokines in the ipsilateral hippocampus, and these responses are significantly reduced in the $\mathrm{p} 38 \alpha \mathrm{KO}$ mice. Specifically, TBI-induced upregulation of the cytokines IL-1 $\beta$, IL-6, 
and TNF $\alpha$ was significantly blunted in the $\mathrm{KO}$ mice (Fig. 2a-c), with a similar trend in IL-33 levels that did not reach significance (Fig. 2d). Similarly, there was a significant reduction in levels of the monocyte recruitment chemokines CCL2 (Fig. 2e) and CXCL10 (Fig. 2f) in the $\mathrm{KO}$ mice after TBI.

Given the significant decrease in multiple pro-inflammatory cytokines as well as chemotactic mediators of monocyte recruitment, we next examined whether $\mathrm{p} 38 \alpha \mathrm{KO}$ mice were spared from TBI-induced monocyte recruitment to the injured brain. We have recently shown that there is a temporally coordinated recruitment of inflammatory monocytes to the brain following TBI that peaks approximately 1 day post-injury $[5,20]$. Therefore, we used this time point to quantify this response using flow cytometry with a combination of antigenic markers designed to delineate activated microglia from infiltrated monocytes. As shown in Fig. 3, we found that the p38 $\alpha$ KO mice have approximately $50 \%$ less infiltration of peripherally derived monocytes into the injured brain, compared to WT mice.
Removal of microglial p38a blunts subacute inflammatory and histopathological responses to TBI

We next examined the consequences of the absence of p38 $\alpha$-driven microglial responses to trauma in the context of subacute inflammatory responses (7 days post-injury). This phase following injury represents a period where peripheral immune infiltration has stopped, and the bulk of inflammatory responses are associated with resident effectors, as we have previously described [24]. Therefore, we tested here the neuroinflammatory responses of WT and $\mathrm{p} 38 \alpha \mathrm{KO}$ mice at 7 days following injury (Fig. 4). At this post-injury interval, IL-1 $\beta$ had returned to approximately basal levels (Fig. 4a), while there was a non-significant trend for IL-6 reduction (Fig. 4b) in the KO mice. The KO mice had significantly blunted pro-inflammatory cytokine response for TNF $\alpha$ (Fig. 4c) and IL-33 (Fig. 4d), as well as a significant reduction in the pro-inflammatory chemokines CCL2 (Fig. 4e) and CXCL10 (Fig. 4f). We also examined the histopathological response of microglia in the dorsal hippocampus at this subacute interval. Using Iba1 as
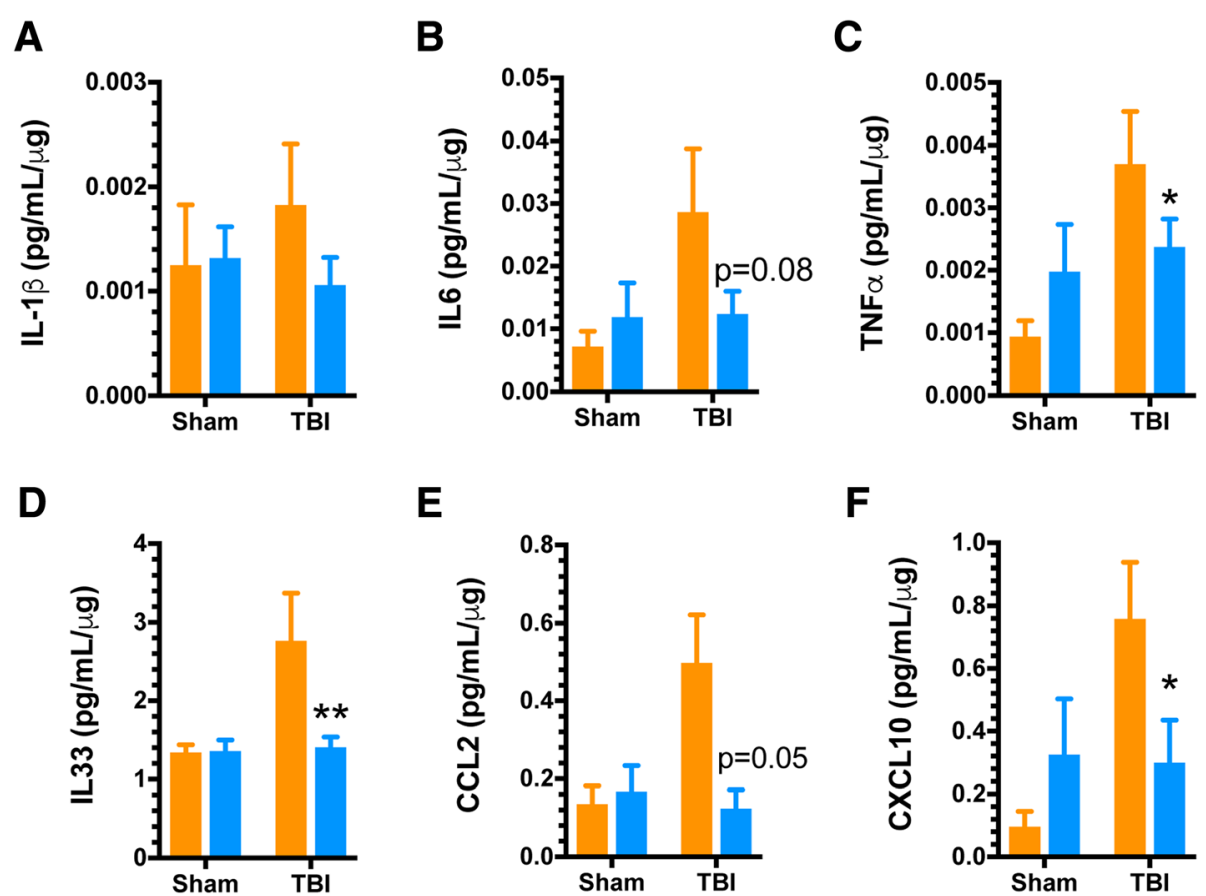

Fig. 4 Persistent pro-inflammatory response is blunted by microglial p38a deletion. Ipsilateral dorsal hippocampi were analyzed via high sensitivity multiplex MSD ELISA assay for pro-inflammatory cytokines and chemokines from sham and injured animals $(n=8-10 / g r o u p)$ at a 7 days post-injury interval. a, bTrauma-induced expression of IL-1 $\beta$ had subsided to approximately basal levels in all groups. There was a persistent trend for increased IL6 production in WT mice that was reduced in KO mice, but the values did not reach significance. $\mathbf{c}, \mathbf{d}$ The chronic elevation of TNFa was significantly reduced in the KO compared to WT mice. The levels of IL-33 remained in an elevated state in WT mice but were significantly reduced in $\mathrm{KO}$ mice. e, f The chronic elevation of the chemokines CCL2 and CXCL10 seen in the WT mice was reduced in the $\mathrm{KO}$ mice. Data were analyzed using two-way ANOVA with Sidak's multiple comparison corrections on the pre-planned contrasts examining the effect of TBI in the WT versus KO conditions. ${ }^{*} p<0.05,{ }^{* *} p<0.01$, comparing KO (blue bars) to WT (orange bars) 


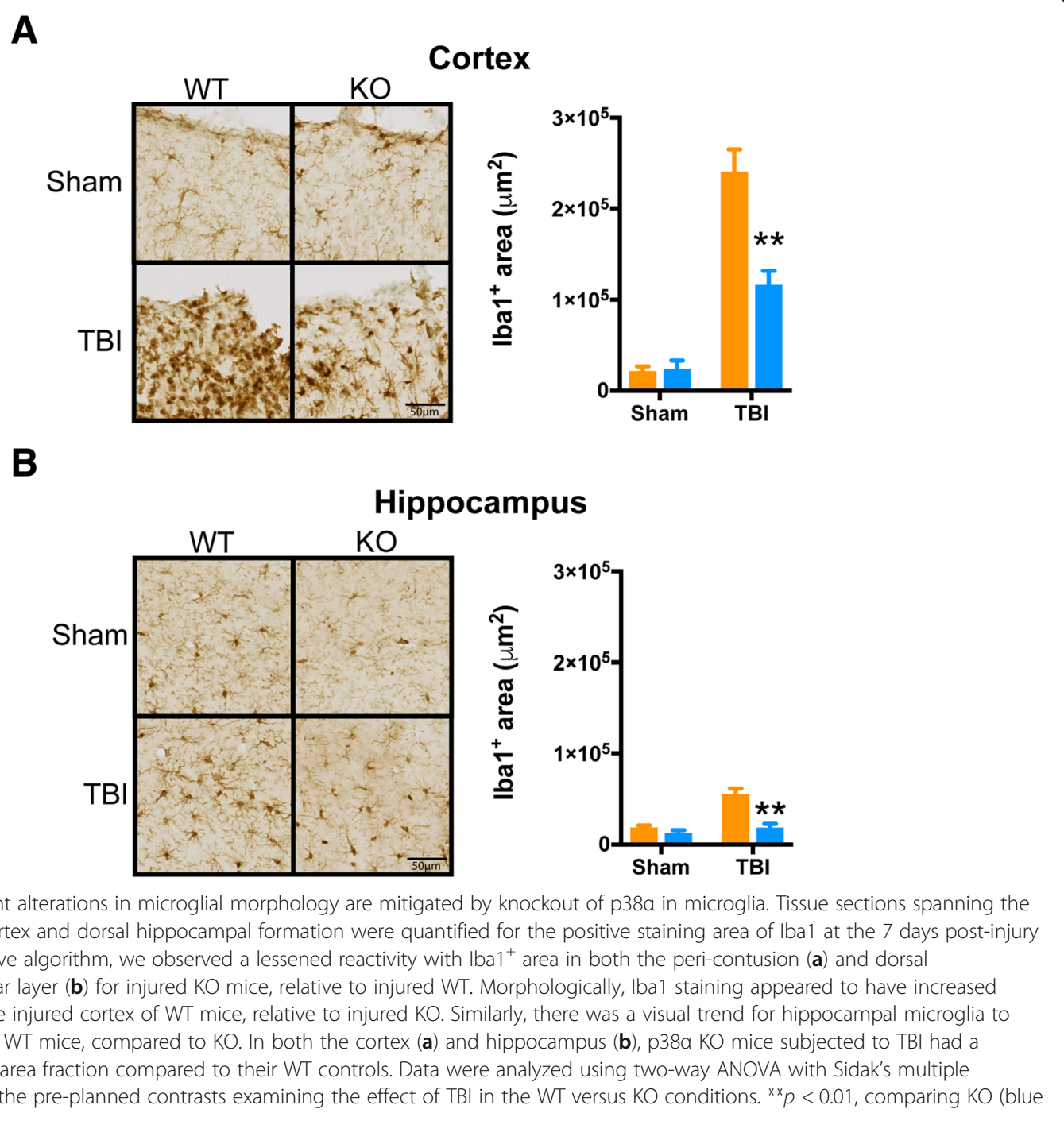

a marker of microglia/macrophage morphology, we observed a protracted increase in Iba1+ area in the peri-contusion cortex and the dorsal hippocampus of WT mice at 7 days post-injury. However, this persistent TBI-induced microglial response was significantly blunted by approximately $50 \%$ in the peri-contusion cortex and entirely absent in the p38 $\alpha \mathrm{KO}$ mouse at this time point (Fig. 5a, b).

\section{Discussion}

In the current study, we have demonstrated a microglia-specific role for $\mathrm{p} 38 \alpha$ in the production of trauma-induced neuroinflammatory sequelae at acute and subacute intervals. We showed that $\mathrm{p} 38 \alpha$ plays an integral role in the production of multiple pro-inflammatory signaling mediators and potentiates the recruitment of inflammatory monocytes to the injured parenchyma. Moreover, our current results demonstrate that deletion of $\mathrm{p} 38 \alpha$ from microglia provides significant amelioration of both acute and protracted trauma-induced inflammatory responses, including accelerating the return of microglia morphological phenotypes towards basal phenotypes. Collectively, these findings further demonstrate that microglial p38 $\alpha$ plays an integral role in propagating trauma-induced neuroinflammatory sequelae.

Microglia have an intrinsic ability to mobilize and rapidly respond to injury $[1,2]$. However, the unabated and/ or chronic activation of these cells is an integral link in the pathophysiological response propagating dysfunction sequelae, in the clinical setting as well as in preclinical animal models of TBI trauma [25-28]. In terms of the involvement of p38-mediated inflammatory responses in CNS trauma, multiple reports have documented a significant role for $\mathrm{p} 38$ following both TBI and spinal cord injury [29-32]. Mice that are deficient in MK2, which is a direct and selective substrate of $\mathrm{p} 38 \alpha$, showed 
significant improvements in multiple measures of recovery following spinal cord injury, compared to WT [30]. Collectively, there is growing data demonstrating a role for $\mathrm{p} 38 \alpha$ and its regulated effectors in promoting dysfunctional responses following CNS trauma.

Our results demonstrate that deletion of $\mathrm{p} 38 \alpha$ in microglia provides significant amelioration of whole-tissue pro-inflammatory cytokines and chemokines and significantly limits the ingress of inflammatory monocytes that we have previously attributed to causing significant detrimental effects upon neuronal and cognitive function [5]. Therefore, our new findings specifically implicate microglial p38 $\alpha$ as an integral mediator creating a permissive environment for the recruitment of neurotoxic monocytes.

Further, the effects of targeting p $38 \alpha$ in microglia provided protracted benefits, as there was a collective mitigation of trauma-induced pro-inflammatory bias in $\mathrm{KO}$ animals relative to their WT counterparts at a subacute 7-day post-injury interval. These effects were seen by the return of microglia morphological response to sham-like levels in the KO mice, compared to WT mice, which still exhibited a perturbed morphological phenotype.

Our current findings warrant extended exploration of the role of $\mathrm{p} 38 \alpha$ in microglia responses to TBI. It still remains unknown if the alterations in pro-inflammatory bias conferred by loss of $p 38 \alpha$ in microglia offer neuroprotective capacities and whether these ultimately offer functional restoration at chronic intervals.

\section{Conclusion}

Restraint of microglia and their inflammatory responses following neurotrauma represents an ever-growing area for targeted therapeutic development. Our current efforts further support the potential benefits of discretely targeting microglia through inhibition of $\mathrm{p} 38 \alpha$, or potentially its downstream intermediates, as a potential strategy to blunt neuroinflammatory sequelae.

\section{Abbreviations}

CCl: Controlled cortical impact; KO: Knockout; MAPK: Mitogen-activated protein kinase; MSD: MesoScale Discovery; PBMCs: Peripheral blood mononuclear cells; PBS: Phosphate-buffered saline; RFP: Red fluorescent protein; TBI: Traumatic brain injury; W: Wildtype

\section{Acknowledgements}

We thank Dr. Huiping Jiang for the kind gift of the floxed p38a mice. Also, we thank Amy Gorman and Edgardo Dimayuga for their excellent technical assistance.

\section{Funding}

Research reported in this publication was supported by the National Institute of Neurological Disorders R01 NS093920 (LJVE). The content is solely the responsibility of the authors and does not necessarily represent the official views of the National Institutes of Health.

\section{Availability of data and materials}

The datasets generated and/or analyzed during this study are available from the corresponding author on reasonable request.

\section{Authors' contributions}

JMM and LJVE designed the research studies. JMM and DSG performed the experiments. JMM and LJVE analyzed the data and wrote the manuscript. All authors read and approved the final manuscript.

\section{Ethics approval}

All procedures involving animals for this study were approved by the Institutional Animal Care and Use Committee of the University of Kentucky.

\section{Consent for publication}

Not applicable.

\section{Competing interests}

The authors declare that they have no competing interests.

\section{Publisher's Note}

Springer Nature remains neutral with regard to jurisdictional claims in published maps and institutional affiliations.

\section{Author details}

${ }^{1}$ Sanders-Brown Center on Aging, University of Kentucky, 101 Sanders-Brown Bldg., 800 S. Limestone Street, Lexington, KY 40536, USA. ${ }^{2}$ Department of Neuroscience, University of Kentucky, Lexington, KY, USA. ${ }^{3}$ Spinal Cord and Brain Injury Research Center, University of Kentucky, Lexington, KY 40536, USA.

Received: 11 February 2019 Accepted: 30 April 2019

Published online: 10 May 2019

\section{References}

1. Davalos D, Grutzendler J, Yang G, Kim JV, Zuo Y, Jung S, Littman DR, Dustin ML, Gan W-B. ATP mediates rapid microglial response to local brain injury in vivo. Nat Neurosci. 2005:8:752-8.

2. Nimmerjahn A, Kirchhoff F, Helmchen F. Resting microglial cells are highly dynamic surveillants of brain parenchyma in vivo. Science (New York, NY). 2005;308:1314-8.

3. Morganti-Kossmann MC, Satgunaseelan L, Bye N, Kossmann T. Modulation of immune response by head injury. Injury. 2007;38:1392-400.

4. Kigerl KA, Gensel JC, Ankeny DP, Alexander JK, Donnelly DJ, Popovich PG. Identification of two distinct macrophage subsets with divergent effects causing either neurotoxicity or regeneration in the injured mouse spinal cord. J Neurosci. 2009;29:13435-44.

5. Morganti JM, Jopson TD, Liu S, Riparip LK, Guandique CK, Gupta N, Ferguson AR, Rosi S. CCR2 antagonism alters brain macrophage polarization and ameliorates cognitive dysfunction induced by traumatic brain injury. J Neurosci. 2015:35:748-60.

6. Donnelly DJ, Longbrake EE, Shawler TM, Kigerl KA, Lai W, Tovar CA, Ransohoff RM, Popovich PG. Deficient CX3CR1 signaling promotes recovery after mouse spinal cord injury by limiting the recruitment and activation of Ly6Clo/iNOS+ macrophages. J Neurosci. 2011;31:9910-22.

7. Gurtner GC, Werner S, Barrandon Y, Longaker MT. Wound repair and regeneration. Nature. 2008;453:314-21.

8. Ramlackhansingh AF, Brooks DJ, Greenwood RJ, Bose SK, Turkheimer FE, Kinnunen KM, Gentleman S, Heckemann RA, Gunanayagam K, Gelosa G, Sharp DJ. Inflammation after trauma: microglial activation and traumatic brain injury. Ann Neurol. 2011;70:374-83.

9. Hernandez-Ontiveros DG, Tajiri N, Acosta S, Giunta B, Tan J, Borlongan CV. Microglia activation as a biomarker for traumatic brain injury. Front Neurol. 2013:4:30.

10. Giunta B, Obregon D, Velisetty R, Sanberg PR, Borlongan CV, Tan J. The immunology of traumatic brain injury: a prime target for Alzheimer's disease prevention. J Neuroinflammation. 2012;9:185.

11. Ziebell JM, Morganti-Kossmann MC. Involvement of pro- and antiinflammatory cytokines and chemokines in the pathophysiology of traumatic brain injury. Neurotherapeutics. 2010;7:22-30.

12. Bachstetter $A D$, Van Eldik $L$. The p38 MAP kinase family as regulators of proinflammatory cytokine production in degenerative diseases of the CNS. Aging Dis. 2010;1:199-211.

13. Bachstetter AD, Rowe RK, Kaneko M, Goulding D, Lifshitz J, van Eldik L. The p38a MAPK regulates microglial responsiveness to diffuse traumatic brain injury. J Neurosci. 2013;33:6143-53. 
14. Orthgiess J, Gericke M, Immig K, Schulz A, Hirrlinger J, Bechmann I, Eilers J. Neurons exhibit Lyz2 promoter activity in vivo: implications for using LysMCre mice in myeloid cell research. Eur J Immunol. 2016;46:1529-32.

15. Schnöder L, Hao W, Qin Y, Liu S, Tomic I, Liu X, Fassbender K, Liu Y. Deficiency of neuronal p38a MAPK attenuates amyloid pathology in Alzheimer disease mouse and cell models through facilitating lysosomal degradation of BACE1. J Biol Chem. 2016;291:2067-79.

16. Gibbs KL, Kalmar B, Rhymes ER, Fellows AD, Ahmed M, Whiting P, Davies $\mathrm{CH}$, Greensmith L, Schiavo G. Inhibiting p38 MAPK alpha rescues axonal retrograde transport defects in a mouse model of ALS. Cell Death Dis. 2018; 9:197.

17. Corrêa SAL, Eales KL. The role of p38 MAPK and its substrates in neuronal plasticity and neurodegenerative disease. J Signal Transduct. 2012;2012:1-12

18. Bruchas MR, Schindler AG, Shankar H, Messinger DI, Miyatake M, Land BB, Lemos JC, Hagan CE, Neumaier JF, Quintana A, et al. Selective p38alpha MAPK deletion in serotonergic neurons produces stress resilience in models of depression and addiction. Neuron. 2011;71:498-511.

19. Yona S, Kim K-W, Wolf Y, Mildner A, Varol D, Breker M, Strauss-Ayali D, Viukov S, Guilliams M, Misharin A, et al. Fate mapping reveals origins and dynamics of monocytes and tissue macrophages under homeostasis. Immunity. 2013;38:79-91.

20. Morganti JM, Riparip LK, Chou A, Liu S, Gupta N, Rosi S. Age exacerbates the CCR2/5-mediated neuroinflammatory response to traumatic brain injury. J Neuroinflammation. 2016;13:80

21. Morganti JM, Riparip LK, Rosi S. Call Off the Dog(ma): M1/M2 polarization is concurrent following traumatic brain injury. PLoS One. 2016;11:e0148001.

22. Cardona AE, Huang D, Sasse ME, Ransohoff RM. Isolation of murine microglial cells for RNA analysis or flow cytometry. Nat Prot. 2006;1:1947-51.

23. Xing B, Bachstetter AD, van Eldik L. Microglial p38a MAPK is critical for LPSinduced neuron degeneration, through a mechanism involving TNFa. Mol Neurodegener. 2011;6:84.

24. Hellewell S, Semple BD, Morganti-Kossmann MC. Therapies negating neuroinflammation after brain trauma. Brain Res. 2015.

25. Kelley BJ, Farkas O, Lifshitz J, Povlishock JT. Traumatic axonal injury in the perisomatic domain triggers ultrarapid secondary axotomy and Wallerian degeneration. Exp Neurol. 2006;198:350-60.

26. Lloyd E, Somera-Molina K, van Eldik L, Watterson DM, Wainwright MS Suppression of acute proinflammatory cytokine and chemokine upregulation by post-injury administration of a novel small molecule improves long-term neurologic outcome in a mouse model of traumatic brain injury. J Neuroinflammation. 2008;5:28.

27. Schmidt Ol, Heyde CE, Ertel W, Stahel PF. Closed head injury--an inflammatory disease? Brain Res Brain Res Rev. 2005;48:388-99.

28. Cao T, Thomas TC, Ziebell JM, Pauly JR, Lifshitz J. Morphological and genetic activation of microglia after diffuse traumatic brain injury in the rat. Neuroscience. 2012;225:65-75.

29. Stirling DP, Liu J, Plunet W, Steeves JD, Tetzlaff W. SB203580, a p38 mitogen-activated protein kinase inhibitor, fails to improve functional outcome following a moderate spinal cord injury in rat. Neuroscience. 2008; 155:128-37.

30. Ghasemlou N, Lopez-Vales R, Lachance C, Thuraisingam T, Gaestel M, Radzioch D, David S. Mitogen-activated protein kinase-activated protein kinase 2 (MK2) contributes to secondary damage after spinal cord injury. J Neurosci. 2010;30:13750-9.

31. Mori T, Wang $X$, Jung J-C, Sumii $T$, Singhal $A B$, Fini ME, Dixon $C E$, Alessandrini A, Lo EH. Mitogen-activated protein kinase inhibition in traumatic brain injury: in vitro and in vivo effects. J Cereb Blood Flow Metab. 2002;22:444-52.

32. Xu Z, Wang B-R, Wang X, Kuang F, Duan X-L, Jiao X-Y, Ju G. ERK1/2 and p38 mitogen-activated protein kinase mediate iNOS-induced spinal neuron degeneration after acute traumatic spinal cord injury. Life Sci. 2006;79:1895-905.

Ready to submit your research? Choose BMC and benefit from:

- fast, convenient online submission

- thorough peer review by experienced researchers in your field

- rapid publication on acceptance

- support for research data, including large and complex data types

- gold Open Access which fosters wider collaboration and increased citations

- maximum visibility for your research: over $100 \mathrm{M}$ website views per year

At $\mathrm{BMC}$, research is always in progress.

Learn more biomedcentral.com/submissions 